\title{
Review
}

\section{Progress in the Research on the Relationship between Infection with Human Papillomavirus and Tumorigenesis}

\author{
Jin Zhang \\ School of Medicine and Life Sciences, Jinan University, Jinan China
}

\author{
Keywords \\ human papillomavirus; infection; tumor; \\ correlation \\ Correspondence \\ Jin Zhang, \\ E-mail: zhangjjn@163.com
}

DOI: 10.1515/ii-2017-0138

\begin{abstract}
Human papillomavirus (HPV) is a DNA virus that infects the skin and mucous membranes of the human body. Approximately $80 \%$ of sexually active women are likely infected with HPV. Cervical cancer is one of the most common malignant tumors and is second in incidence only to breast cancer. Infection with high-risk HPV types is the main risk factor for cervical cancer, which is currently the only malignant tumor with a clearly defined etiology. HPV infection is also closely related to the incidence and development of other malignant tumors. In addition to cervical cancer, HPV can cause other urogenital tumors, as well as tumors in the digestive tract, lungs, eyes, skin, and other organ systems. This paper provides a review of the progress in HPV infection-related research and provides novel ideas for the study of tumor etiology and mechanisms.
\end{abstract}

Human papillomavirus (HPV) is a DNA virus that infects the basal epithelium of the skin and mucous membranes of the human body. It is ubiquitous among the population but its incidence is highest among sexually active young individuals. Approximately 200 types of HPV have been identified. These HPV types are categorized as high-risk or low-risk types based on their pathogenicity. The 13 highrisk HPV types are HPV16, 18, 31, 33, 35, 39, 45, 51, 52, 56, $58,59 \mathrm{~m}$, and 68 . HPV 16 and HPV18 account for $50 \%$ to $60 \%$ of HPV cases, and are the most closely related with the incidence of malignant tumors. The risk of HPV infection is associated with age, gender, number of sexual partners, and other factors. The carcinogenic mechanism of HPV remains unclear and likely involves a multi-factorial, multistep progressive process. Continuous, high viral load is the necessary condition for HPV-induced tumorigenesis. More than $10 \%$ of malignant tumors are related to HPV infection. Early studies have shown that HPV DNA is integrated into the host cell chromosome and interferes with the expression of vascular endothelial growth factor (EGFR) through E2 gene inactivation and the E5 gene, thus up-regulating the expression of the E6 and E7 proteins. The E6 and E7 proteins respectively inhibit the activity of
P53 and RB genes, activate the transcription of human cell telomerase, cause the abnormal differentiation of cells, lead to the immortalization of normal cells, and finally initiate tumorigenesis. Nevertheless, the immortalization of HPVinduced host cells cannot be explained merely by the lack of E2, the overexpression of E6 and E7, and host gene stability. In addition, integrated viral genes are not present in all HPV-related malignant tumors. Epidemiological studies have shown that some infections are associated with oralgenital contact. Viruses spread to other mucous membranes and initiate subclinical infection. After activation by certain unknown mechanisms, viruses will cause cancer and initiate malignant transformation. HPV infection has become a necessary condition for cervical cancer and may be a risk factor for other primary or secondary tumors.

\section{HPV and urogenital malignant tumors}

\section{Cervical cancer}

Cervical cancer is the cancer with the second highest incidence in women, and is closely related with HPV. Approximately $80 \%$ of sexually active women may be 
infected with HPV. Each year, there are approximately 500,000 new cases of cervical cancer in the world and approximately 250,000 individuals die of cervical cancer. Studies have shown that HPV is present in tissue samples from $99.7 \%$ of cervical cancer cases. HPV16 and HPV18 account for $70 \%$ of cancer cases, and $50 \%$ of cervical cancer cases is caused by HPV16. HPV 6, 44, 53, and 54 appear in multiple infections, whereas all other HPV types are single infection. Infection with a single low-risk HPV type has not been found in cervical cancer tissues. High-risk HPV types are co-infected with low-risk HPV types. HPV16 is most closely related with cervical squamous carcinoma and HPV18 most likely causes cervical adenocarcinoma. Epidemiological research and evidence have identified HPV as the cause of cervical cancer. The cancerization of cells by HPV is caused by multiple factors that interact with each other. However, not all HPV infections will develop into cancer: the carcinogenesis of HPV is dependent on the body's immunity and persistent HPV infection.

\section{Ovarian cancer}

Ovarian cancer is closely related with HPV16 and HPV18, and is positively correlated with vascular endothelial growth factor ki-67. In 2012, Malisice et al. ${ }^{[2]}$ studied 54 patients with ovarian cancer. They identified four cases of positive HPV16 infection based on the results of PCR detection and the clinicopathological features of postoperative tissue. They found that HPV DNA existed in $7.4 \%$ of the tissue, indicating that HPV may be related to the occurrence of ovarian cancer. In addition, the rate of HPV16 infection in ovarian cancer tissue was significantly higher than that in normal ovarian tissue. These results implicate HPV as the cause of ovarian cancer.

\section{Vulvar and vaginal cancer}

Domestic and foreign studies have shown that HPV DNA is present in the majority of vulvar and vaginal cancer patients. In 2008, Sutton et al. ${ }^{[3]}$ studied paraffin-embedded tissue sections from 116 cases of vulvar squamous cell carcinoma and found $81 \mathrm{HPV}$-positive cases, among which HPV16 was the most common type of infection. DeVuyst et al. ${ }^{[4]}$ detected HPV DNA in $40.4 \%$ and $69.9 \%$ of 1873 cases of vulvar cancer and 136 cases of vaginal cancer, respectively. HPV 16 accounted for $75.0 \%$ of the detected cases, and its detection rate was $5 \%$ more than that of cervical cancer. Therefore, HPV infection likely plays an important role in the development of vulvar and vaginal cancers. In addition, infection with high-risk HPV types may be a risk factor for these two kinds of cancers.

\section{Penile cancer}

$\mathrm{HPV}$ is closely related to penile cancer. Infection with HPV16 is the most common type of HPV infection among penile cancer cases and might also be a cause of penile cancer. In 2009, Miralles-Guri et al. ${ }^{[5]}$ provided a systematic and comprehensive review of 1466 penile cancer cases from 1986 to 2008 . They found that $46.9 \%$ of cases were infected with HPV. Among these cases, infection with HPV16 was the most common (60.2\%), followed by HPV 18 (13.3\%). Calmon et al. ${ }^{[6]}$ detected HPV DNA in $48.9 \%$ of 47 specimens of penile squamous cell carcinoma. Most detected HPV types were high-risk HPV (42.5\%). Therefore, high-risk HPV plays an important role in the development and progression of penile cancer.

\section{Breast cancer}

HPV16 and HPV18 can promote the immortalization of normal human breast epithelial cells. Possible infection pathways of HPV in the breast include: 1) Transfer of HPV from the genital tract to the blood and (or) lymphatic metastasis. 2) Invasion of HPV through sexual activity. 3) Invasion of HPV along mammary ducts, thus inducing the carcinogenesis of terminal lobular units or ductal epithelial basal stem cells. Researchers have extensively studied the presence of HPV in breast cancer tissue but reported significantly different results and correlations. However, with the development of various detection technology, many reports have implied that HPV exists in breast cancer tissue. Pereira et al. ${ }^{[7]}$ analyzed 61 cases of fresh frozen breast cancer specimens and reported that 16 , or $26 \%$, of these case were $\mathrm{HPV}$ positive

\section{Bladder cancer}

The relationship between HPV and bladder cancer has drawn increased attention along with the development of PCR technology, which has enabled the growth of relevant research on HPV. A meta-analysis by Chinese scholars of literature on HPV and bladder cancer from the past 10 
years reported that $35.60 \%$ of Asian patients with bladder cancer were infected with HPV; $34.29 \%$ of these cases were infected with high-risk HPV types. Moreover, $47.20 \%$ of Chinese mainland patients with bladder cancer were infected with HPV ${ }^{[8]}$. Zhu Weizhi et al. ${ }^{[9]}$ used secondgeneration hybrid capture technology to test 65 cases of bladder transitional cell carcinoma and 30 cases of normal bladder tissue. They found that high-risk HPV16/18 was present in 42 cases of bladder cancer tissue at a positive rate of $64.60 \%$. High-risk HPV 16/18 was also found in two cases of normal bladder tissue with a positive rate of $6.70 \%$. This result indicated that the detection rate of highrisk HPV16/18 in in bladder cancer tissue was significantly higher than that in normal bladder tissue. Therefore, HPV16/18 infection may be involved in the incidence of bladder transitional cell carcinoma and likely affects the malignancy grade and progression of bladder cancer.

\section{HPV and gastrointestinal malignant tumors}

\section{Oral cancer}

The occurrence of oral cancer is a multi-step, multi-factorial complex process. In recent years, studies have found that the occurrence of oral squamous cell carcinoma is closely related with high-risk HPV16 and HPV18 infection ${ }^{[10]}$; that the number of vaginal intercourse and oral sex partners has increased among oral cancer patients; that marijuana users are mostly HPV-positive; and that HPV-positive patients have better prognosis. Babiker et al. ${ }^{[11]}$ screened 200 cases of oral disease patients for HPV and reported that high-risk HPV infection accounted for $6 \%$, or 12 , cases. Among these 12 cases, eight were oral cancer cases and four were nonneoplastic lesion cases, in which HPV16 accounted for 50\%. These results suggested that high-risk HPV is a risk factor for oral cancer.

\section{Esophageal cancer}

Esophageal cancer is one of the ten most common malignant tumors in China. Many scholars have begun to study the effect of HPV infection on esophageal epithelial cell carcinoma. Their results showed that in different regions, the positive HPV rate ranged between $0 \%$ and $67 \%$. Dabrowski et al. ${ }^{[12]}$ performed quantitative DNA analysis in 56 cases of esophageal cancer and 56 cases of normal mucosal tissue from patients in southeastern Poland. Their results showed that $50 \%$, or 28 cases, of esophageal cancer patients were positive for HPV DNA. Among these cases, five were positive for HPV16 and HPV18, accounting for $8.9 \%$ of cases. Only four cases in the normal mucosa group were positive for HPV DNA. No high-risk types were detected. The incidence of HPV in esophageal cancer was significantly higher than that in the control group.

\section{Gastric cancer}

Gastric cancer originates in gastric mucosa epithelial cells and is related with HPV infection. Chinese scholars used in situ PCR and immunohistochemistry to detect E6, the HPV16 cancer gene, in tissue samples from gastric cancer patients in Shaanxi Province. They detected HPV16 E6 in $25 \%(11 / 40)$ of 40 cases of gastric cancer but not in 40 cases of corresponding non-tumor healthy tissue. In addition, the infection rate of HP V16 in cardiac carcinoma was significantly higher than that in non-cardiac cancer ${ }^{[13]}$.

\section{Colorectal cancer}

HPV infection was firstly assumed to be associated with squamous cell carcinoma. However, later studies reported that adenocarcinoma might also be associated with HPV infection because HPV DNA was first detected in tissue from cecal adenocarcinoma cases. Using PCR analysis to analyze the relationship between HPV infection and colorectal cancer, Ma Heng et al. ${ }^{[14]}$ detected HPV in 135 cases of colorectal cancer and in 25 cases of normal colorectal mucosa. Their results showed that the overall HPV positive rate of colorectal cancer was $32.59 \%$, which was significantly higher than that in normal mucosa tissue. Most cases were infected with HPV16, followed by HPV18. At the same time, they confirmed that HPV infection was unrelated with the location of colorectal cancer, pathological grade, clinical stage, and lymph node metastasis.

\section{Anal cancer}

The incidence of anal cancer is low compared with those of other cancers but has increased in recent years. Most cases of anal cancer are associated with HPV, especially HPV16 ${ }^{[15]}$. Varnai et al. ${ }^{[16]}$ detected HPV DNA by using PCR and found that $80.9 \%$ of anal cancer cases were positive for HPV. 
This result indicated that high-risk HPV infection could increase the risk of anal cancer.

\section{HPV and respiratory tract malignant tumors}

\section{Nasopharyngeal carcinoma}

Via fluorescence quantitative PCR detection, Huang Zhongqiang et al. ${ }^{[17]}$ found that out of 150 cases of nasopharyngeal carcinoma, 55 cases (36.7\%) were positive for HPV16 and HPV18. Out of 50 cases of normal healthy people, three cases (6\%) were positive for HPV16 and HPV18. The detection rate of HPV in the case group was significantly different from that in the control group, indicating that HPV16 and HPV18 are closely related with the incidence and development of nasopharyngeal cancer and may even cause this type of cancer.

\section{Tonsil cancer}

Maier et al. ${ }^{[18]}$ studied specimens from 223 cases of head and neck tumors. The HPV detection rate in tonsil cancer cases was $37.5 \%$, ranking the first. These cases were mainly infected with HPV16, thus implicating HPV infection in tonsil cancer. Studies have also shown that patients with HPV-positive tonsil cancer and tongue cancer have better prognosis ${ }^{[19]}$.

\section{Laryngeal cancer}

By using in situ hydbridization, Jiangyin Rong et al. ${ }^{\text {[20] }}$ detected HPV16 and HPV18 mRNA in laryngeal mucosa from 123 cases of laryngeal squamous cell carcinoma and 123 cases of healthy individuals. The HPV16 E6 and HPV18 E6 positive rates were $46.34 \%$ and $35.77 \%$, respectively. The high-risk HPV positive rate was considerably higher than that in the normal group, indicating that HPV infection is an important risk factor for laryngeal cancer. The retention and sustained expression of the E6 fragment is closely related with the carcinogenesis of laryngeal tissue.

\section{Lung cancer}

Lung cancer is one of the most serious malignant tumors that endanger human health. Many scholars believe that $\mathrm{HPV}$ is a risk factor for lung cancer ${ }^{[21]}$. Kong Linghui et al. ${ }^{[22]}$ used specific PCR detection to detect HPV16 and
HPV18 DNA in 47 cases of non-small cell lung cancer and in 13 cases of benign lung lesions. They detected HPV16 and HPV18 DNA in $42.55 \%$ (20/47) of the non-small cell lung cancer group. This detection rate was significantly higher than the detection rate of $7.69 \%(1 / 13)$ in the benign lung lesions group. Thus, HPV infection exists in lung cancer tissue.

\section{HPV and ophthalmic malignant tumors}

\section{Conjunctival cancer}

HPV is implicated in the development of conjunctival cancer via interaction with UV or other factors. Payayed et al. ${ }^{[23]}$ detected HPV DNA in $92.9 \%$ of conjunctival tissue from conjunctival cancer cases. Most scholars believe that HPV may be associated with conjunctival cancer but that HPV alone is not carcinogenic and must work with other factors to cause conjunctival cancer.

\section{Retinoblastoma}

Retinoblastoma is a malignant tumor produced by the loss of function of the tumor suppressor gene; this loss-of-function is caused by a mutation in the $\mathrm{Rb}$ gene. HPV infection is a risk factor for retinoblastoma. Animal experiments showed that transgenic mice expressing HPV16 E6 and E7 can develop neuroblastoma. Montoya et al. ${ }^{[24]}$ detected HPV DNA in $82.3 \%$ of five cases of hereditary binocular retinoblastoma. By contrast, 7 out of 10 randomly selected cases were negative for HPV.

\section{HPV and skin cancer}

Skin cancer is divided into two categories: melanoma and non-melanoma. The most common categories of melanoma are malignant melanoma, squamous cell carcinoma, and basal cell carcinoma. Karagas et al. ${ }^{[25]}$ detected HPV DNA in 252 cases of squamous cell carcinoma, 525 cases of basal cell carcinoma, and 461 control cases. They found that DNA levels in the squamous cell carcinoma group were significantly higher than those in the control group were not significantly different between the basal cell carcinoma and control groups. The HPV positive rate was $19 \%$ to $58 \%$, with high-risk HPV types being dominant. These results 
indicated that malignant skin tumors might be associated with HPV infection.

\section{Summary}

Tumorigenesis is a multi-step process that is caused by the interaction of multiple factors. Persistent HPV infection is a direct cause of cervical cancer, and is correlated with tumorigenesis in other organ systems. Although relevant studies were conducted in different geographical locations and reported different specimen sources and detection methods, research results had shown that HPVrelated tumors are mainly caused by high-risk HPV types, especially HPV16. However, the close relationship between HPV infection and tumorigenesis needs further study. The correlation of HPV with tumor development in other organ systems will clarify tumorigenesis and broaden research ideas for tumor treatment.

\section{Declarations}

\section{Acknowledgements}

No.

\section{Competing interests}

The author declares that he has no competing interest.

\section{Authors' contributions}

J Zhang made the literature analysis and wrote, discussed and revised the manuscript of this review.

\section{References}

1 Saavedra KP, Brebi PM, Roa JC. Epigenetic alterations in pre-neoplastic and neoplastic lesions of the cervix. Clin Epigenetics, 2012, 4(1): 13.

2 Malisic E, Jankovic R, Jakovljevic K. Detection and genotyping of human papillomaviruses and their role in the development of ovarian carcinoma. Arch Gynecol Obstet, 2012, 286(3): 723-728.

3 Sutton BC, Allen RA, Moore WE, et al. Distribution of human papillomavirus genotypes in adult squamous carcinoma of the vulva. Mod Pathol, 2008, 21(3): 345-354.

4 De Vuyst H, Clifford GM, Nascimento MC, et al. Prevalence and type distribution of human papillomavirus in carcinoma and intra- epithelial neoplasia of the vulva, vagina and anus: a meta-analysis. Int J Cancer, 2009, 124(7): 1626-1636.
5 Miralles-Guri C, Bruni L, Cubilla AL, et al. Human papilloma- virus prevalence and type distribution in penile carcinoma. J Clin Pathol, 2009, 62(10): 870-878.

6 Calmon MF, Mota MT, Babeto E, et al. Overexpression of ANXA1 in penile carcinomas positive for high-risk HPVs. PLoS One, 2013, 8(1): e53260.

7 Pereira SAL, Lorenzetti MA, Gonzalez LR, et al. Presence of human papillomavirus in a series of breast carcinoma from Argentina. PLoS One, 2013, 8 (4): e61613.

8 Yang L, Li N, Chen Y, et al. Meta-analysis of human papillomavirus infection and risk of bladder cancer in Asia. Chinese Journal of Cancer, 2011, 20(8): $573-578$.

9 Zhu W, Yao X, Wen $\mathrm{H}$, et al. The relationship between bladder cancer and human papillomavirus type 16/18 infection. Chinese Journal of Hospital Infection, 2013, 23(10): 2282-2301.

10 Chaturvedi P, Vaishampayan SS, Nair S, et al. Oral squamous cell carcinoma in background of oral submucous fibrosis: A clinic pathologically distinct disease. Head Neck, 2013, 35(10): 1404-1409.

11 Babiker AY, Eltom FM, Abdalaziz MS, et al. Screening for high risk human papilloma virus (HR-HPV) subtypes, among $\mathrm{Su}$ - danese patients with oral lesions. Int J Clin Exp Med, 2013, 6(4): 275-281.

12 Dabrowski A, Kwasniewski W, Skoczylas T, et al. Incidence of human papilloma virus in esophageal squamous cell carcinoma in patients from the Lublin region. World J Gastroenterol, 2012, 18(40): 5739-5744.

13 Liu W, Zhang M, Chu Y, et al. Study on the correlation between HPV16 and Helicobacter pylori infection in gastric. Modern Journal of Oncology, 2009, 17(6): 1012-1016.

14 Ma H, Cai S, Li Y, et al. Detection of HPV gene in colorectal cancer. Chinese Journal of Clinical Oncology, 2003, 30(2): 16-18, 22.

15 Cornall AM, Roberts JM, Garland SM, et al. Anal and perianal squamous carcinomas and high-grade intraepithelial lesions exclusively associated with "low-risk" HPV genotypes 6 and 11. Int J Cancer, 2013, 133(9): 2253-2258.

Varnai AD, Bollmann M, Griefingholt $\mathrm{H}$, et al. HPV in anal squamous cell carcinoma and anal intraepithelial neoplasia (AIN). Impact of HPV analysis of anal lesions on diagnosis and prognosis. Int J Colorectal Dis, 2006, 21(2): 135-142.

17 Huang Z, Wei X. Detection of HPV16 and 18 in nasopharyngeal carcinoma in Northwest China and its clinical significance. Chinese Journal of Practical Medicine, 2010, 5(13): 84-85.

18 Maier M, Kraft K, Steinestel K, et al. Human papillomavirus in squamous cell cancer of the head and neck: A study at the Ulm Military Hospital, Germany. HNO, 2013, 61(7): 593-601.

19 HPV-positive tonsil cancer and tongue cancer patients with better prognosis. Pharmacy Progress 2008, 32(7): 335

20 Jiang H, Peng F, Chen Y, et al. Expression of HPV-16E6 and HPV-18E6 
in laryngeal squamous cell carcinoma by in situ hybridization. Journal of Immunology, 2009, 25(4): 442-445.

21 Guo F, Fan Y, Qiao Y, et al. Research progress on the correlation of HPV and lung cancer. Chinese Journal of Lung Cancer, 2012, 15(3): 191-194.

22 Kong L, Wu C, Li Y, et al. Expression of E2F-1 and survivin protein in non-small cell lung cancer and their correlation with HPV infection. Chinese Journal of Cancer, 2011, 21(2): 81-85.

23 Moubayed P, Mwakyoma H, Schneider DT. High frequency of human papillomavirus $6 / 11,16$, and 18 infections in precancerous lesions and squamous cell carcinoma of the conjunctiva in subtropical Tanzania. Am J Clin Pathol, 2004, 122(6): 938- 943.

24 Montoya-Fuentes $\mathrm{H}$, de la Paz Ramirez-Munoz M, Villar-Calvo V, et al. Identification of DNA sequences and viral proteins of 6 human papillomavirus types in retinoblastoma tissue. Anti-cancer Res, 2003, 23(3C): 2853-2862.

25 Karagas MR, Nelson $\mathrm{HH}$, Sehr P, et al. Human papillomavirus infection and incidence of squamous cell and basal cell carcinomas of the skin. J Natl Cancer Inst, 2006, 98(6): 389-395. 